\title{
PALLIATIVE CARE IN CANCER MANAGEMENT
}

Cancer care has been transformed in the past 30 years from diagnosis and advanced therapeutics to holistic care. However, it still largely remains a chronic progressive illness, especially in the developing world. The present-day comprehensive cancer care includes integration between active anticancer therapies along with supportive care. Even in the third world, it is an imperative that parallel with anticancer therapies, maintaining quality of life and dignity of patient should be in primary focus.

A multidisciplinary approach in cancer care is essential, and thus, the concept of palliative care should be introduced as early as possible after diagnosis. ${ }^{[1]}$ The patient should not hence feel abandoned as the input from palliative care increases and that of oncology goes down.

Dedicated palliative care units can provide expertise in palliative intervention such as symptomatic control and comprehensive end of life care. Oncologists can admit patients to these units for the management of symptoms, respite, complication of therapy and terminal care. ${ }^{[2]}$

Palliative care should not be synonymised with end of life care only. Management of acute symptoms in cancer patients at various stages of their disease is of paramount importance for physical and emotional well-being of the patient. Good symptomatic control of disease can result in better compliance.

Pain is the most common symptom in cancer that may occur at various stages of disease. A comprehensive evaluation of pain and its management is essential for a good quality of life. This can be a mixture of analgesics as a first step, leading to more complex interventions such as nerve blocks and targeted radionuclide therapies.

Palliative medicine is still in its infancy in Pakistan. Although it is a part of curriculum of postgraduate training in oncology, there are only handful cancer centres, which benefit from a dedicated palliative care service.

Shaukat Khanum Memorial Cancer Hospital and Research Centre has taken the National lead in establishing a physician led palliative care service to support cancer care in the hospital. There is a dedicated in-patient unit with regular multidisciplinary clinics.

Infrequent availability of opioids, compared to some parts of the world, continues to be a major hurdle in delivering and developing palliative care services in Pakistan. The medical community, especially the oncologists, needs to take this issue as a top priority. We need to advocate for the inclusion and delivery of palliative care modules in undergraduate as well as postgraduate curricula.

Haroon Hafeez

Palliative Care Consultant, Department of Internal Medicine, Shaukat Khanum Memorial Cancer Hospital and Research Centre, Lahore, Pakistan Received: 31 August 2015 / Accepted: 10 September 2015

\section{References}

1. Morita T, Akechi T, Ikenaga M, et al. Communication about ending of anticancer treatment and transition to palliative care. Ann Oncol 2004;15:1551-7.

2. Hanks G, Cherny NI, Christakis NA, et al. Oxford Textbook of Palliative Medicine. $4^{\text {th }}$ ed. Oxford, UK: Oxford University Press; 2010.

\footnotetext{
Correspondence: Haroon Hafeez, Palliative Care Consultant, Department of Internal Medicine, Shaukat Khanum Memorial Cancer Hospital and Research Centre, Lahore, Pakistan.

Email: haroonh@skm.org.pk
} 